\title{
Very high energy emission sources beyond the Galaxy
}

\author{
V.G. Sinitsyna ${ }^{a}$, M.S. Andreeva, K.A. Balygin, S.S. Borisov, I.A. Ivanov, A.M. Kirichenko, A.I. Klimov, I.P. Kozhukhova, \\ R.M. Mirzafatikho, N.I. Moseiko, I.E. Ostashev, A.I. Palamarchuk, V.Y. Sinitsyna, and I.G. Volokh
}

P. N. Lebedev Physical Institute, Russian Academy of Science, Russia

\begin{abstract}
Active Galactic Nuclei (AGN) are considered as potential extragalactic sources of very and ultra high energy cosmic rays. According to theoretical predictions cosmic ray acceleration can take place at the shock created by the expanding cocoons around active galactic nuclei as well as at AGN jets. The measurements of AGN TeV spectra, the variability time scale of $\mathrm{TeV}$ emission can provide essential information on the dynamics of AGN jets, the localization of acceleration region and an estimation of its size. SHALON observations yielded data on extragalactic sources of different AGN types in the energy range of $800 \mathrm{GeV}-100 \mathrm{TeV}$. The data from SHALON observations are compared with those from other experiments at high and very high energies.
\end{abstract}

\section{Introduction}

High and very high energy $\gamma$-rays have come to play an important role in the study of Active Galactic Nuclei (AGN). Many AGNs have been detected from $\mathrm{MeV}$ to $\mathrm{TeV}$ energies. The detection of any flares from AGNs in VHE gamma-rays and the determination of the time-scales of the flux variability in different wavelengths, including TeV energies, helps to clarify the location of the emission and limits the size of the emitting region.

The long term SHALON observations yielded data on AGNs of different types in the energy range of 800 $\mathrm{GeV}$ - $100 \mathrm{TeV}$ (Table 1). Some of these AGNs were discovered at $\mathrm{TeV}$ energies by SHALON. The results on fluxes, spectral energy distributions and images are shown here and in [1-7]. All SHALON results are compared with data from experiments at high and very high energies (see Figs. 1,2,3,4).

\section{Mkn $421(\mathrm{z}=\mathbf{0 . 0 3 1})$, Mkn 501 $(\mathrm{z}=0.034)$ and Mkn $180(\mathrm{z}=0.046)$}

The observation results of known BL Lacs Mkn 421, Mkn 501 and Mkn 180 are presented (see Fig. 2, [7]). The images and spectral energy distributions at energies above $800 \mathrm{GeV}$ are obtained for each of the sources.

A number of flare periods in different wavelengths including VHE $\gamma$-rays were found. For example, the last flaring state of Mkn 501 at $\mathrm{TeV}$ energies was detected by SHALON between March and June 2009. A flux increase was detected on 23-24 April and 23-25 May with an average flux of $(3.41 \pm 0.70) \times 10^{-12} \mathrm{~cm}^{-2} \mathrm{~s}^{-1}$. The increase of the Mkn 501 flux in the 2009 SHALON data correlates with one at a lower energy range of $>$ $2 \mathrm{GeV}$ by Fermi LAT and has a time lag with the flare at 0.2-2.0 GeV [8]. Also, this flare was detected at $\mathrm{TeV}$ energies by VERITAS, MAGIC and Whipple [8].

a e-mail: sinits@sci.lebedev.ru
Mkn 180 was observed by SHALON in 2007, 20092014, for a total of 71 hours. After the standard analysis, a clear excess corresponding to an $18.5 \sigma$ effect [9] was detected. The Mkn 180 spectrum fitting parameters are shown in Table 1.

Extreme variability in different wavelengths, including VHE $\gamma$-rays, on time-scales from minutes to years is the most distinctive feature of BL Lac objects, but the variations in total optical brightness are small. Also, no evidence for any significant flux variability was found during the full observation period of Mkn180 by SHALON.

\section{3 C $382(\mathrm{z}=\mathbf{0 . 0 5 7 8})$}

3C 382 is a typical Broad Line Radio Galaxy (BLRG), essentially identical to a quasar except for the optical luminosity. 3C382 is a well-known X-ray source. Its radio structure shows several quasar-like features. The core is very bright and there is a narrow jet with a compact hotspot and with a lobe opposite the jet. In contrast to blazars, the jets in BLRGs do not point directly toward the observer, and the relativistic beaming effects and the related jet dominance are only moderate, so it becames possible to investigate both the accretion disk and the jet. Our observations are mainly aimed at searches for $\mathrm{TeV} \gamma$ ray emission from $3 \mathrm{C} 382$.

3C 382 was detected by SHALON at TeV energies [4] (in observations during the years 2009-2016, 46.3 hours in total) with a significance [9] of $9.5 \sigma$. The integral $\gamma$-ray flux above $0.8 \mathrm{TeV}$ was estimated to be $(0.91 \pm$ $0.14) \times 10^{-12} \mathrm{~cm}^{-2} \mathrm{~s}^{-1}$. The energy spectrum of $\gamma$-rays at energies above $800 \mathrm{GeV}$ is described by a power law with index $-1.08 \pm 0.11$. Correlations of the $\mathrm{TeV}$ flux with $\mathrm{MeV}-\mathrm{GeV}$ energy fluxes were not found as the observations of 3C 382 at high energies with Fermi LAT [10] only gave an upper limit in the range 0.1-100 GeV (Fig. 2).

(C) The Authors, published by EDP Sciences. This is an Open Access article distributed under the terms of the Creative Commons Attribution License 4.0 (http://creativecommons.org/licenses/by/4.0/). 
Table 1. The catalogue of extragalactic $\gamma$-ray sources observed by SHALON with parameters for spectrum fitting in the form of a power low with exponential cutoff $F(>E) \propto E^{k_{\gamma}} \times \exp \left(-E / E_{\text {cutoff }}\right)$.

\begin{tabular}{cccccc}
\hline Sources & Observable flux $^{a}$ & $k_{\gamma}$ & $E_{\text {cutoff }, \mathrm{TeV}}$ & Distance, z & Type \\
\hline NGC 1275 & $(0.78 \pm 0.05)$ & $-2.18 \pm 0.12$ & $32 \pm 7$ & 0.018 & Seyfert \\
SN2006 gy & $(3.71 \pm 0.65)$ & $-3.10 \pm 0.30$ & $4.4 \pm 1.9$ & 0.019 & SN \\
IC 310 & $(0.89 \pm 0.09)$ & $-0.84 \pm 0.10$ & $13 \pm 3.9$ & 0.019 & RG \\
Mkn 421 & $(0.63 \pm 0.05)$ & $-1.51 \pm 0.18$ & $10 \pm 3$ & 0.031 & BL Lac \\
Mkn 501 & $(0.86 \pm 0.06)$ & $-1.48 \pm 0.15$ & $11 \pm 3$ & 0.034 & BL Lac \\
Mkn 180 & $(0.65 \pm 0.09)$ & $-1.84 \pm 0.15$ & $7.3 \pm 2.2$ & 0.046 & BL Lac \\
3c382 & $(0.91 \pm 0.14)$ & $-1.05 \pm 0.11$ & $21 \pm 7.0$ & 0.0578 & BLRG \\
4C+31.63 & $(0.73 \pm 0.16)$ & $-1.13 \pm 0.16$ & $10.4 \pm 3.2$ & 0.295 & FSRQ \\
OJ 287 & $(0.26 \pm 0.07)$ & $-1.14 \pm 0.11$ & $9.5 \pm 1.2$ & 0.306 & BL Lac \\
3C 454.3 & $(0.43 \pm 0.07)$ & $-0.52 \pm 0.12$ & $6.2 \pm 1.0$ & 0.859 & FSRQ \\
4C+55.17 & $(0.90 \pm 0.16)$ & $-1.40 \pm 0.15$ & $5.4 \pm 2.1$ & 0.896 & FSRQ \\
PKS 1441+25 & $(0.52 \pm 0.23)$ & - & - & 0.939 & FSRQ \\
1739+522 & $(0.49 \pm 0.05)$ & $-0.50 \pm 0.18$ & $6.1 \pm 1.2$ & 1.375 & FSRQ \\
B2 0242+43 & $(0.58 \pm 0.20)$ & - & - & 2.243 & FSRQ \\
B2 0743+25 & $(0.37 \pm 0.16)$ & - & - & 2.979 & FSRQ \\
\hline
\end{tabular}

${ }^{a}$ Integral flux at energy $>800 \mathrm{GeV}$ in units of $10^{-12} \mathrm{~cm}^{-2} \mathrm{~s}^{-1}$.
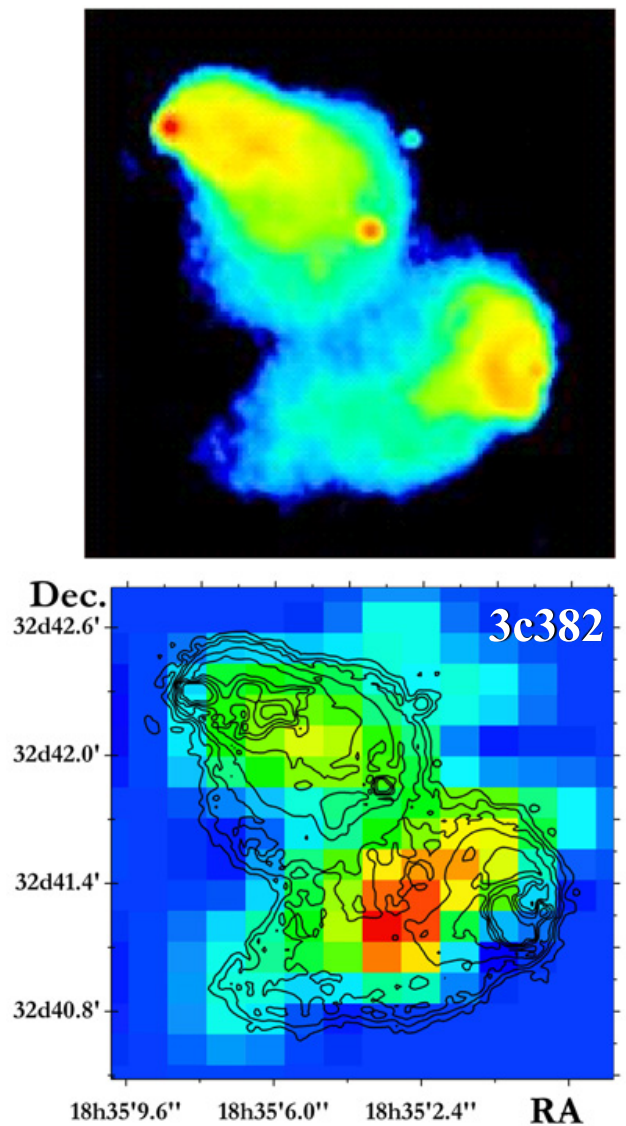

Figure 1. Top: VLA radio image of of 3c382. Bottom: SHALON image of $3 \mathrm{c} 382$ at energies $800 \mathrm{GeV}-35 \mathrm{TeV}$. The contours represent the source's radio structure from VLA.

Figure 1 presents a VLA $1477 \mathrm{MHz}$ image of 3C 382. It shows a core-lobe structure with hot-spots at the end of the North jet and at the South. The TeV image of 3C 382 is correlated with the structures visible in radio energies by VLA [11]. The structure viewed in TeV $\gamma$-rays by SHALON (Fig. 1) can be the result of heating or blowing the interstellar medium by jets similar to one observed in the NGC 1275 Seyfert galaxy. No significant emission was found from the position of core core, which is clearly seen in the radio-image.

\section{OJ $287(\mathrm{z}=\mathbf{0 . 3 0 6})$}

OJ 287 is a low-frequency peaked BL Lac object. It is one of the most studied blazars whose spectrum has been well measured through radio $[12,13]$ to $\mathrm{X}$-ray bands $[14,15]$ and for optical studies. The most outstanding characteristic of OJ 287 is its 12 year period, which has been found in the optical [14] and also confirmed in X-rays. OJ 287 is supposed to be a binary black hole system in which a secondary black hole passes the accretion disk of the primary black hole and produces two impact flashes per period. The spectral energy distributions of blazars consist of two broad peaks. The first, lower frequency peak, is due to the synchrotron emissions of relativistic electrons in the jet. It is supposed that the second, higher frequency peak, is due to Inverse Compton emission of the same electrons [16]. GeV emission from OJ 287 has been detected by EGRET [17] and Fermi LAT [18]. It has also been suggested to be a TeV source.

OJ 287 was observed by SHALON in 1999, 2000, 2008-2010, for a total of 47.3 hours, at zenith angles ranging from $22^{\circ}$ to $34^{\circ}$. The observations of 1999 and 2000 do not reveal a $\gamma$-ray flux from the position of OJ 287 , but only an upper limit of $<1.1 \times 10^{-13} \mathrm{~cm}^{-2} \mathrm{~s}^{-1}$. In the observations of 2008, 2009 and 2010 (31.2 hours) a weak $\gamma$-ray flux was detected [3]. An excess corresponding to a $6.9 \sigma$ effect was observed. The measured integral energy spectrum of OJ 287 can be well described by a power law with index $-1.43 \pm 0.18$. The observed integral flux above $800 \mathrm{GeV}$ is $(0.26 \pm 0.07) \times 10^{-12} \mathrm{~cm}^{-2} \mathrm{~s}^{-1}$. The image of $\gamma$-ray emission from OJ 287 by SHALON telescope is shown in Fig. 2.

A flux increase over the detected average flux was found on 14, 15 November and 4, 5 December 2010. The average value of the increase is $(0.63 \pm 0.15) \times$ $10^{-12} \mathrm{~cm}^{-2} \mathrm{~s}^{-1}$ (statistical significance of $6.2 \sigma$ [9]) with a softer energy spectrum with a power law of index $-1.96 \pm 0.16$. A high flux was also detected on 16 

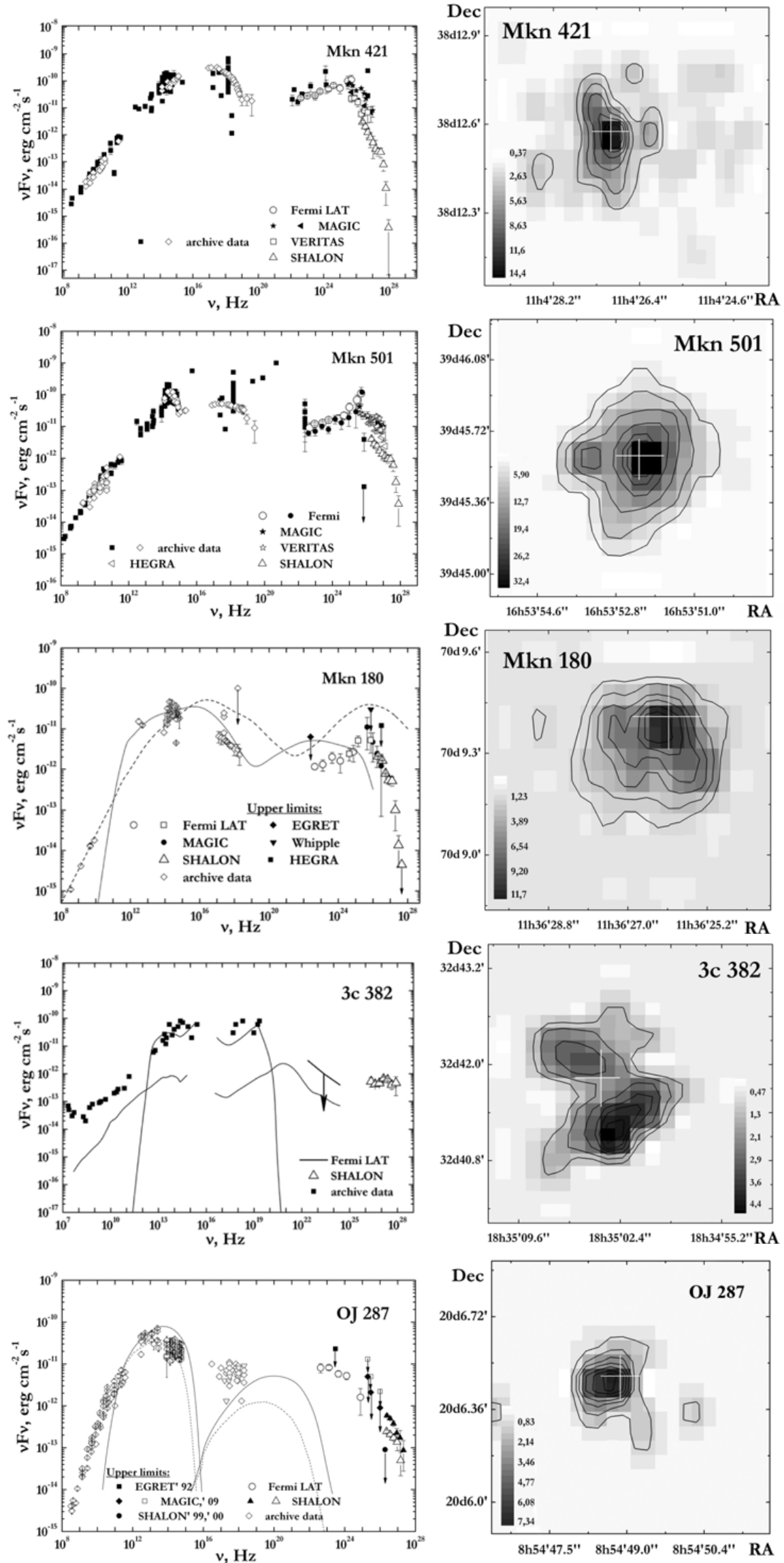

Figure 2. Spectral energy distributions (left) and images (right) of AGNs by SHALON compared with other experiments. 


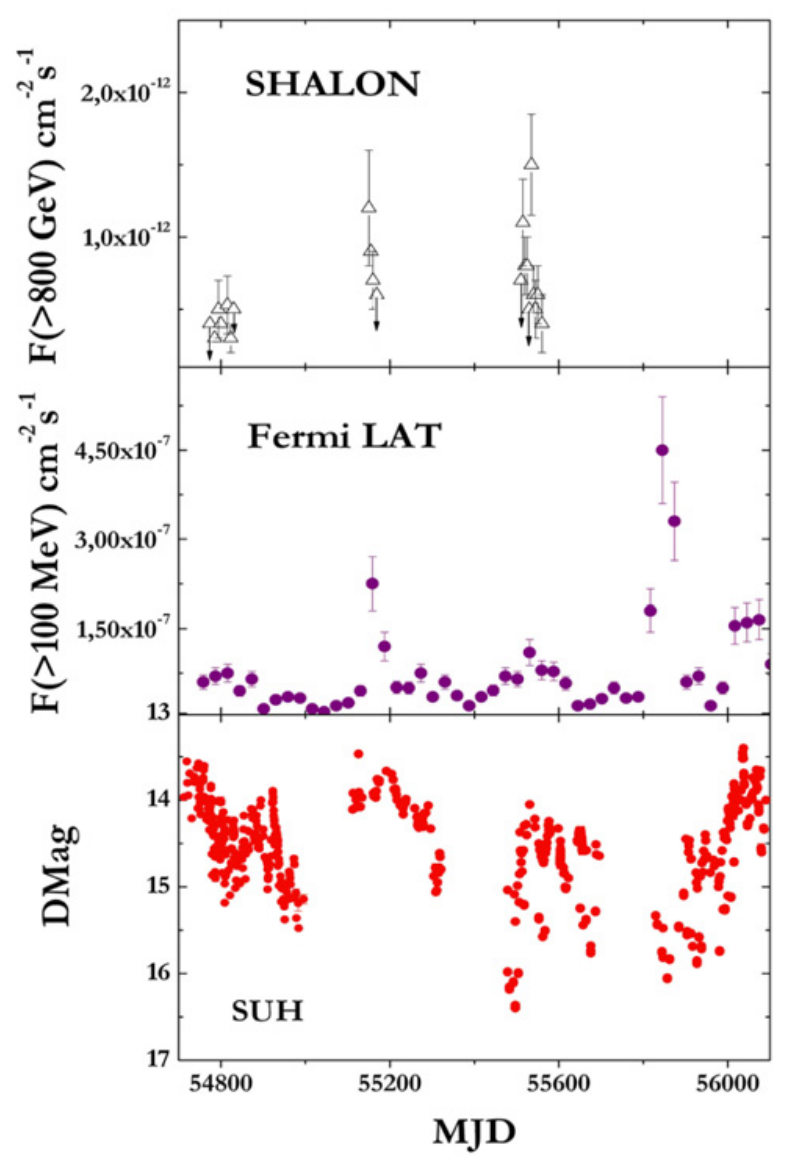

Figure 3. Comparison of the OJ 287 light curve observed by SHALON in the energy range $800 \mathrm{GeV}-35 \mathrm{TeV}$ with data in the MeV-GeV energies by Fermi LAT and in R-band.

November 2009. The duration of the flux increase in December 2010 was 2 days with a peak intensity $(1.16 \pm$ $0.51) \times 10^{-12} \mathrm{~cm}^{-2} \mathrm{~s}^{-1}$. No duration of intervals of flux increase were found in 2009 , because the observations were interrupted due to weather conditions.

Figure 2 presents the spectral energy distribution of the $\gamma$-ray emission from OJ 287 by SHALON in comparison with other experiments' data - MAGIC [19], EGRET [17], and with theoretical predictions from [18] and [19]. The $\triangle$ at $\mathrm{TeV}$ energies in Fig. 2 are the SHALON spectrum of OJ287; an upper limit at $>0.8 \mathrm{TeV}$ corresponds to SHALON observations in 1999, 2000. The $\boldsymbol{\Delta}$ present the $\gamma$-ray spectrum during the increased flux period of 2010 . OJ 287 is the weakest extragalactic source observed by SHALON.

To reveal possible correlations of the emissions in various energy ranges, including those at high and very high energies, we compared the OJ287 $\gamma$-ray fluxes observed by SHALON in the periods when the observations were simultaneous with those by the Fermi LAT experiment (see Fig. 3) and data in R-band (Suhora Astronomical Observatory) [20]. A slight local flux increase can be seen in the period of November 2010 in the Fermi LAT data [21] and at R-band, which corresponds to the above-mentioned $\gamma$-ray flux increase observed by SHALON in 2010. The increase of OJ 287 flux in the 2009 SHALON data correlates with flares at MeV-GeV energies and R-band (see Fig. 3).
In [22] it is shown that the GeV flares are produced due to the interaction of moving plasma blobs with a standing shock. The flare correlations can indicate that the TeV emission observed by SHALON is a very high energy population of particles formed in the spectrum viewed by Fermi LAT, which are produced by the synchrotron selfCompton process at the jet away from the central core [22].

\section{5. $4 \mathrm{C}+31.63(\mathrm{z}=0.295)$}

To collect data for FSRQ type, sources at a large distance range from $\mathrm{z}<0.3$ to extremely high $\mathrm{z}$ sources from the first and second Fermi LAT AGN catalogue are observed by SHALON.

$4 \mathrm{C}+31.63$ is a FSRQ type source at redshift $\mathrm{z}=$ 0.295 and was observed by SHALON in the period 2012 2015 for a total of 45.7 hours. $\gamma$-ray emission from the position of $4 \mathrm{C}+31.63$ was detected above $800 \mathrm{GeV}$ [1] with a $9.7 \sigma$ effect. Energy spectrum parameters are shown in Table $1.4 \mathrm{C}+31.63$ was previously detected at high energies with Fermi LAT. The image of $4 \mathrm{C}+31.63 \mathrm{in} \mathrm{TeV}$ $\gamma$-rays by the SHALON telescope is shown in Fig. 4. No high activity of $4 \mathrm{C}+31.63$ in $\mathrm{TeV}$ energies was found.

\section{6. $3 C 454.3(\mathrm{z}=\mathbf{0 . 8 5 9})$}

3C 454.3 is a known flat-spectrum radio quasar at redshift $\mathrm{z}=0.859$. It has shown very high activity over the last fifteen years and displayed variability at all wavelengths. 3C 454.3 was detected at high energies by the EGRET telescope, with an average photon index of $\Gamma=2.2$. 3C 454.3 was detected with Fermi LAT at high energies in 2008 [23]. This object has been particularly bright in the $\gamma$-ray band covered by AGILE and Fermi LAT.

In 1998 3C 454.3 was detected by SHALON at TeV energies [5] and was observed by SHALON in the period from 1998 to 2013, for a total of 69.2 hours, at zenith angles ranging from $18^{\circ}$ to $35^{\circ}$. After the standard analysis, a clear excess corresponding to a $14.9 \sigma$ effect [9] was determined. The observed energy spectrum parameters and integral $\gamma$-ray flux are presented in Table 1 . The $\mathrm{TeV}$ $\gamma$-ray emission map is presented in Fig. 4 right. The spectral energy distribution of 3C 454.3 and limits on the intrinsic spectrum at $\mathrm{TeV}$ energies de-absorbed using extragalactic background light shapes (see [6]) is presented in Fig. 4 left with lines. 3C 454.3 has been detected with Fermi LAT at energies $200 \mathrm{MeV}-300 \mathrm{GeV}$ [23]. The spectrum by Fermi LAT is fitted with a broken powerlaw with photon indices $\Gamma_{\text {low }}=2.27 \pm 0.3, \Gamma_{\text {high }}=3.5 \pm$ 0.05 with an average flux of $\sim 3 \times 10^{-6} \mathrm{~cm}^{-2} \mathrm{~s}^{-1}$, for energies $>100 \mathrm{MeV}$. Additional identification of detected sources with 3C 454.3 is performed because of the correlation of high level $\mathrm{TeV}$ fluxes with its flares at lower energies.

3C 454.3 shows a significant flux variability in the different energy ranges including high and very high energies. A number of flaring periods in observations of 3C 454.3 were detected by SHALON since 1998 . A period of high level TeV flux was detected in 2006 from 20 to 28 September. A factor two increase of the TeV flux was detected on 15 November 2009. The flare of 2009 detected by Fermi LAT [24] has a $\sim 10$ day time lag with one at TeV energies. The last significant flaring state of $3 \mathrm{C} 454.3$ at $\mathrm{TeV}$ energies was detected in the SHALON observational 

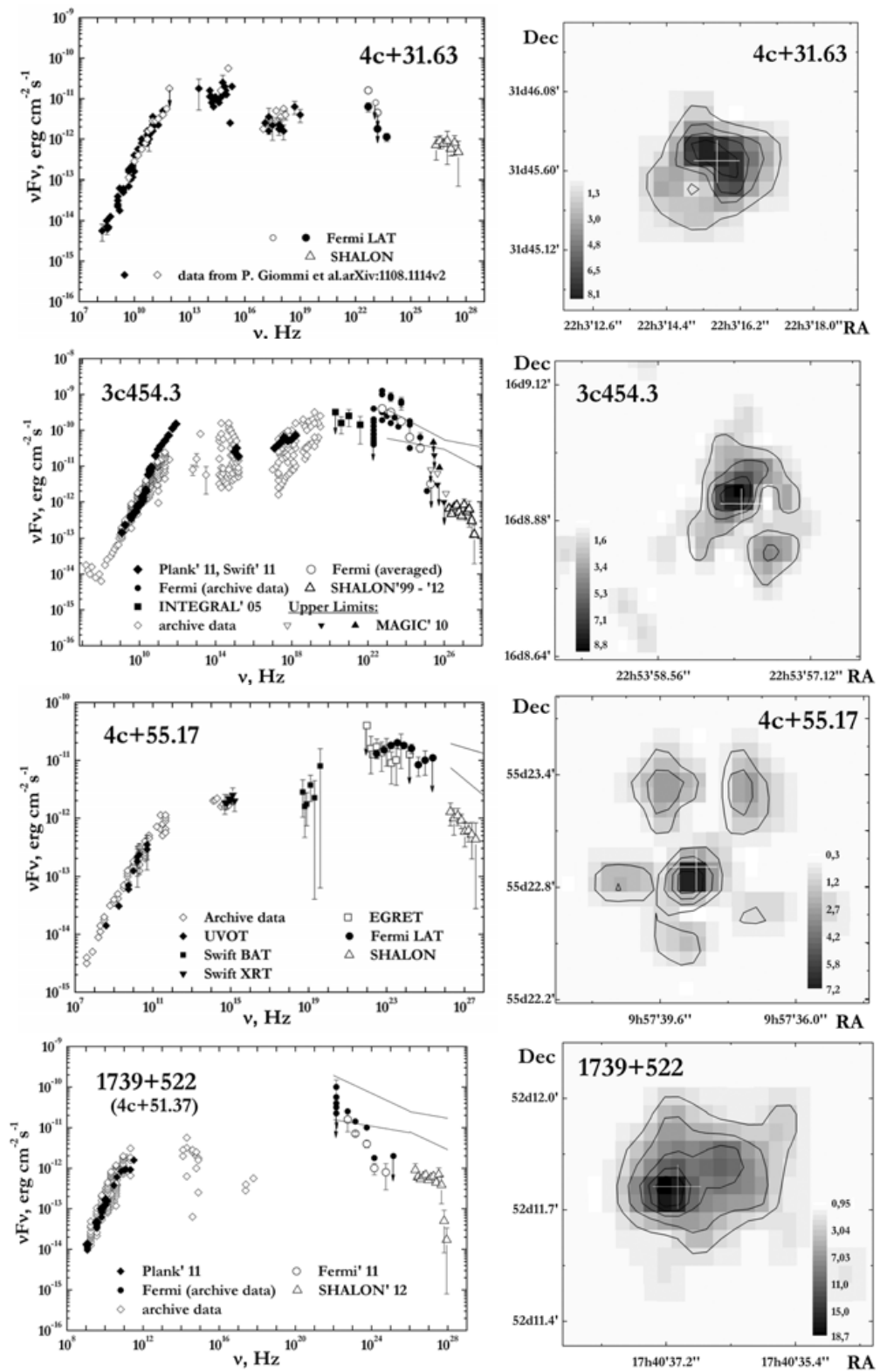

Figure 4. Spectral energy distributions (left) and images (right) of the $\gamma$-ray emission from AGNi observed by SHALON.

period of Nov.-Dec. 2010. The flare of TeV flux detected by SHALON on 10 Nov. 2010 correlates with a flux increase observed by Fermi LAT at the phase of the plateau and flare in R-band. The flux increase was detected on 2-4 Dec. with flux of $(3.41 \pm 0.70) \times 10^{-12} \mathrm{~cm}^{-2} \mathrm{~s}^{-1}$. This increase correlates with flares observed by Fermi LAT [25] and corresponds to the phase of increasing flux of the post-flare.

Very high energy flux variation time-scales observed by SHALON are similar to ones observed by Fermi LAT [24]. The short flaring times suggest parsec-scale size regions for the origin of $\mathrm{TeV}$ and $\mathrm{MeV}-\mathrm{GeV}$ emission [24].

\section{7. $4 \mathrm{C}+55.17(\mathrm{z}=\mathbf{0 . 8 9 6})$}

$4 \mathrm{C}+55.17$ is a radio-loud active galaxy classified as a FSRQ. This object was previously detected at high energies with EGRET and Fermi LAT with an unusually hard MeV-GeV $\gamma$-ray spectrum [26]. 4C + 55.17 was observed by SHALON in the period from 2012-2016 for a total of 61.2 hours [1]. After the standard analysis, a clear excess corresponding to an $8.4 \sigma$ effect [9] was determined with an integral flux and the observed energy spectrum shown in Table 1 . Figure 4 shows the spectral energy distribution of $4 \mathrm{C}+55.17, \triangle$ represent the data from the SHALON telescope, and the line limited area at $\mathrm{TeV}$ energies is the intrinsic spectrum in the source corrected on the extragalactic background light attenuation [6]. The image of $4 \mathrm{C}+55.17$ obtained by SHALON at $\mathrm{TeV}$ energies is in Fig. 4.

It was shown in [26] and [27] that $4 \mathrm{C}+55.17$ demonstrates morphological, spectral properties of FSRQtype blazars. The variability and flaring activity in different wavelengths including $\mathrm{MeV}-\mathrm{GeV}$ energies and $\mathrm{TeV} \gamma$-rays is the most distinctive feature of blazar-type objects. But no evidence for flux variability was found during the full period of observations of $4 \mathrm{C}+55.17$ by SHALON at TeV energies. Also, no evidence of high energy flux variability was found by Fermi LAT [26]. The detection of steady high and very high energy emission in $4 \mathrm{C}+55.17$ together 
with low variability in radio [28] points out an absence of features providing high activity at all wavelengths.

\section{8. $1739+522(4 \mathrm{C}+51.37)(\mathrm{z}=1.375)$}

One more remote metagalactic $\gamma$-source of the FSRQ type was detected by SHALON in 1999 and is being intensively studied since then $[1,5]$. This object was identified with $1739+522(4 \mathrm{C}+51.37)$. It was observed by SHALON in the period from 1999, 2003-2013 for a total of 119.8 hours. The average value of the integral flux is $(0.49 \pm 0.05) \times 10^{-12} \mathrm{~cm}^{-2} \mathrm{~s}^{-1}$ at $E>0.8 \mathrm{TeV}$, with $15.1 \sigma$ [9]. The energy spectrum parameters are shown in Table 1. The image of $4 \mathrm{C}+51.37$ at $\mathrm{TeV}$ energies by SHALON is presented in Fig. 4 ; $\triangle$ on the spectral energy distribution (fig. 4) represent the data from the SHALON telescope. Lines at $\mathrm{TeV}$ energies limit the range of the intrinsic spectrum in the source corrected on the extragalactic background light attenuation [6].

$1739+522$ is a high polarization radio quasar. It is detected by EGRET with an average $\gamma$-flux in the range $\sim 30 \mathrm{MeV}$ to $50 \mathrm{GeV}$ about $2 \times 10^{-8} \mathrm{~cm}^{-2} \mathrm{~s}^{-1}$ with a photon index of $\Gamma \sim 2.2$ [29]. The $100 \mathrm{MeV}-100 \mathrm{GeV}$ $\gamma$-ray emission from $1739+522$ was detected by Fermi LAT with average flux of $F(>1 \mathrm{GeV})=(2.5 \pm 0.2) \times$ $10^{-9} \mathrm{~cm}^{-2} \mathrm{~s}^{-1}$ and power-law with photon index $\Gamma=$ $2.5 \pm 0.04$ [30].

Two periods of $\mathrm{TeV}$ flux increase were found since the first detection of $1739+522$. In observations from 8 to 20 June 2004 the flux increase from the level of the average value up to $(1.93 \pm 0.60) \times 10^{-12} \mathrm{~cm}^{-2} \mathrm{~s}^{-1}$ at $E>0.8 \mathrm{TeV}$ in the peak on 12 June 2004 was recorded in two night observations. A similar high flux increase was detected on 12 May 2005, but no duration of flux increase were found, because the observations were interrupted due to weather conditions.

\section{B2 $0242+43$ and B2 $0743+25(z>2)$}

It was discussed in [31] and later, that the $\gamma$-ray spectra of distant sources at $10 \mathrm{~s}$ of $\mathrm{GeV}$ to $\mathrm{TeV}$ energies begin to be attenuated by the still poorly known EBL photon field due to the $\gamma+\gamma \rightarrow e^{+} e^{-}$process. So, to estimate the cosmic $\gamma$-ray horizon two extremely high-redshifted sources from the first and second Fermi LAT AGN catalogue were observed by SHALON in the autumn-winter period of 2014.

B2 $0242+43$, with $z=2.243$, is a radio-loud active galaxy classified as a FSRQ. It was observed by SHALON in 2014 , for a total of 14 hours, at zenith angles from $16^{\circ}$ to $33^{\circ}$. After the standard analysis, an excess corresponding to a $4.8 \sigma$ effect [9] was determined with an integral flux of $(0.58 \pm 0.20) \times 10^{-12} \mathrm{~cm}^{-2} \mathrm{~s}^{-1}$. The unexpected high-level flux increase up to twice that detected on average was observed at the end of November. The area around B2 $0242+43$ of $20^{\prime}$ size in radius doesn't contain any candidate for TeV-emission nor any GRBs, supernova explosions or other powerful sources over the wide wavelength range variable sources that could be sources of the flares detected in the observation period. The list of nearby sources includes two weak radioobjects and one X-ray source weren't confirmed after their detection $[32,33]$.
B2 $0743+25$, at redshift $\mathrm{z}=2.979$, is an FSRQ from the Fermi LAT catalogues. It was observed by SHALON in the period from 2014 to 2016 on clear moonless nights, for a total of 20 hours, at zenith angles ranging from $15^{\circ}$ to $32^{\circ}$. The integral $\gamma$-ray flux of $I(>0.8 \mathrm{TeV})=$ $(0.37 \pm 0.16) \times 10^{-12} \mathrm{~cm}^{-2} \mathrm{~s}^{-1}$ was determined with a significance of $5.1 \sigma$ [9]. An analysis of the region of $20^{\prime}$ in radius around $\mathrm{B} 20743+25$ was performed in order to find any close powerful sources that could be resolved as this distant quasar $[33,34]$. Among the nearby sources no candidate for $\mathrm{TeV}$-emission were found as four quasar objects, known only due to identification by $\mathrm{V}$ B U magnitude, were not recognized in radio, X-ray or MeV$\mathrm{GeV}$ energies [34].

Further observations and investigations of B2 $0242+$ 43 , B2 $0743+25$ and the area around these objects are continuing to establish a reliable source of $\mathrm{TeV} \gamma$-ray emission.

\section{Conclusion}

The results presented here are a brief summary of longterm observations of AGNs by the SHALON telescope such as spectral energy distributions and images. A number of variability periods at different wavelengths including VHE $\gamma$-rays were found. Short flaring times of very high energy emission from 3C 454.3 and $1739+522$ are found and it suggests parsec scale size regions of $\mathrm{TeV}$ gammaray origin in these sources. In addition, all data obtained in SHALON observations are compared with those from other experiments at high and very high energies.

\section{References}

[1] V.G. Sinitsyna and V.Yu. Sinitsyna "Astroparticle, Particle, Space Physics and Detectors Physics Applications" (Proc. 14th ICATPP, 2013) ed. S. Giani, C. Leroy, P.-G. Rancoita, L. Price and R. Ruchti (Singapore: World Scientific 2014) vol. 8, p. 3 ; ibid. p. 20

[2] V.G. Sinitsyna,V.Yu. Sinitsyna, Astron. Lett. 40 (2-3), 75 (2014)

[3] V.G. Sinitsyna et al., In Proc. of 32nd ICRC, Beijing, China, 2011; vol. 8/11, p. 26

[4] V.G. Sinitsyna, V.Yu. Sinitsyna EPJ Web of Conferences 52, 10005 (2013)

[5] V.G. Sinitsyna et al., Int. J. Mod. Phys. A 29, 7023, 7026, 7029 (2005)

[6] V.G. Sinitsyna et al., J. Phys.: Conf. Ser. 409, 012113 (2013)

[7] V.G. Sinitsyna et al., J. Phys.: Conf. Ser. 409, 012112 (2013)

[8] A.A. Abdo et al., Astrophys. J. 727, 129 (2011)

[9] T.-P. Li and Y.-Q. Ma, Astrophys. J. 272, 317 (1983)

[10] J. Kataoka et al. Astrophys. J. 2011, 740, 29

[11] J.P. Leahy, R.A. Perley Astrophys. J. 102, 537 (1991)

[12] E. Idesawa et al. Publ. Astron. Soc. Jpn. 49, 631 (1997)

[13] H. Seta et al. Publ. Astron. Soc. Jpn. 61, 1011 (2009)

[14] E. Valtaoja et al., Astrophys. J. 531, 744 (2000)

[15] C.E. Tateyama et al. Astrophys. J. 608, 149 (2004)

[16] G. Ghisellini et al. Mon. Not. R. Astron. Soc. 301, 451 (1998)

[17] R.C. Hartman et al., ApJS 123, 79 (1999) 
[18] A.A. Abdo et al., Astrophys. J. 700, 597 (2009); ibid. 715, 429132 (2010)

[19] M. Hayashida et al., In Proc. of 31st ICRC, Lodz, Poland, 2009

[20] P. Pihajoki et al. Astrophys. J. 764, 5 (2013)

[21] M. Ackermann et al. Astrophys. J. 810, 14 (2015)

[22] I. Agudo et al. Astrophys. J. Lett. 726, L13 (2011)

[23] A.A. Abdo et al., Astrophys. J. 699, 817 (2009)

[24] M. Ackermann et al. Astrophys. J. 721, 1383 (2010)

[25] A.A. Abdo et al. Astrophys. J. 733, L26 (2011)
[26] W. McConville et al. Astrophys. J. 738, 148 (2011)

[27] A.P. Marscher et al. Astrophys. J. 577, 85 (2002)

[28] P. Augusto et al. MNRAS 368, 1411 (2006)

[29] R. Mukherjee et al. Astrophys. J. 490, 116 (1997)

[30] P.L. Nolan et al. ApJS 199, 31 (2012)

[31] A.I. Nikishov Sov. Phys. JETP 14, 393 (1962)

[32] I.I.K. Pauliny-Toth et al., Astron. J. 77, 265 (1972)

[33] W. Voges et al. Astron. Astrophys. 349, 389 (1999)

[34] M.-P. Veron-Cetty, P. Veron Astron. Astrophys. 518, A10-10 (2010) 